\title{
Quasi-Adiabatic Continuation in Gapped Spin and Fermion Systems: Goldstone's Theorem and Flux Periodicity
}

\author{
M. B. Hastings \\ Center for Nonlinear Studies and Theoretical Division, \\ Los Alamos National Laboratory, \\ Los Alamos, NM, 87545 \\ hastings@lanl.gov
}

\begin{abstract}
We apply the technique of quasi-adiabatic continuation to study systems with continuous symmetries. We first derive a general form of Goldstone's theorem applicable to gapped nonrelativistic systems with continuous symmetries. We then show that for a fermionic system with a spin gap, it is possible to insert $\pi$-flux into a cylinder with only exponentially small change in the energy of the system, a scenario which covers several physically interesting cases such as an $s$-wave superconductor or a resonating valence bond state.
\end{abstract}


Systems with a spin gap are believed to have a number of interesting properties. One expects these systems to have short-range spin correlations, but in return exotic properties such as fractionalization and topological order often appear[1]. In this paper, we apply the recently developed technique of quasi-adiabatic continuation[2] to study these systems, generalizing ideas developed in [3]. We start by proving a version of Goldstone's theorem, demonstrating exponential decay of spin correlations in a gapped system with continuous symmetries. This result is stronger than previous results [4, 5, 6] which proved exponential decay of connected correlation functions in gapped systems, as in this case we also show that for a system with multiple ground states the average of the spin correlations over different ground states is exponentially small.

We then consider the problem of flux quantization in electron systems with a spin gap (without requiring that there be any gap to spinless excitations). We consider a system of spinful fermions which is periodic in at least one direction, and show that if the system has a spin gap then there is a state which has an expectation value of the energy for a Hamiltonian with $\pi$ flux which is close to the ground state energy of the Hamiltonian with no flux[7]. This result covers various interesting physical cases such as superconductors[8] and resonating valence bond states [9].

Throughout, we consider local Hamiltonians on a lattice as defined below. We begin by defining some notation and defining conditions for a Hamiltonian to be local. Then we review the technique of quasi-adiabatic continuation, and give a brief discussion of Berry's phase for this continuation. We then consider Goldstone's theorem and the flux periodicity problem.

We use labels $i, j, \ldots$ to denote lattice sites, and introduce a metric $\operatorname{dist}(i, j)$. We assume that there is a finite dimensional Hilbert space on each site. An example of a local Hamiltonian is a Hamiltonian of the form $\mathcal{H}=\sum_{i} H_{i}$, where each $H_{i}$ has bounded operator norm: $\left\|H_{i}\right\| \leq J$ for some $J$, and where each $H_{i}$ has support on a set of sites within some distance $R$ of site $i$. This includes lattice spin and fermion models with finite range interactions.

In general, we define a Hamiltonian to be local as follows: we write the Hamiltonian as $\mathcal{H}=\sum_{Z} h_{Z}$ where $Z$ are different sets of sites on the lattice and $h_{Z}$ is a hermitian operator with support on set $Z$. For a Hamiltonian to be local, we require that there exist constants 
$\mu, s_{1}$ such that for all $i$,

$$
\sum_{Z \ni i}\left\|h_{Z}\right\||Z| \exp [\mu \operatorname{diam}(Z)] \leq s_{1}<\infty
$$

where $|Z|$ is the total number of sites within set $Z$ (the cardinality). For Goldstone's theorem we also impose an additional finite dimensionality requirement, Eq. (21), discussed below.

Eq. (10) implies a Lieb-Robinson bound [5, 6, 10]: there exists a velocity $v$ and constant $c$ such that for any two operators $A, B$ defined with support on sets $X, Y$,

$$
\|[A(t), B]\| \leq c \times|X|\|A\|\|B\| \exp [-\mu \operatorname{dist}(X, Y)](\exp [v \mu|t|]-1)
$$

where $A(t)=\exp [i \mathcal{H} t] A \exp [-i \mathcal{H} t]$. This bound is useful for $|t| \leq l / v$, and implies that for any $\mu^{\prime} \leq \mu$, we have

$$
\|[A(t), B]\| \leq c \times|X|\|A\|\|B\| \exp \left[-\mu^{\prime}(\operatorname{dist}(X, Y)]\right.
$$

so long as $t \leq l / v^{\prime}$, with

$$
v^{\prime}=v \mu /\left(\mu-\mu^{\prime}\right)
$$

We will use the bound in the form (3) throughout.

Finally, we emphasize that our results are derived for finite volume systems. The bounds that we find, however, for Goldstone's theorem, do not depend on volume, while we will explicitly discuss the volume dependence for the flux quantization result. Stronger Goldstone bounds may be possible in some cases in the infinite volume limit, as there exist some systems for which, for example, there is a degenerate ground state for a finite size system but a unique ground state in the infinite system[11]; we do not consider these possibilities further. There also exist cases in which there are two approximately degenerate low energy states, which become exactly degenerate in the thermodynamic limit, with a gap to the rest of the spectrum[12]; our results are applicable to this case, as in the proof of the Goldstone theorem we do not require exact degeneracy of the low-lying states.

\section{QUASI-ADIABATIC CONTINUATION}

\section{A. Main Results}

The technique of quasi-adiabatic continuation [2, 13] is a general technique for describing how the ground state of gapped Hamiltonians evolves under a change in a parameter. We 
consider a parameter dependent Hamiltonian $\mathcal{H}_{s}=\sum_{Z} h_{Z}(s)$ for some parameter $s$. We assume the ground state or set of ground states is given at $s=0$ and we wish to describe the evolution of this state or set of states under change in $s$. We denote the ground states of $\mathcal{H}_{s}$ by $\Psi_{0}^{a}(s)$ where $a=1 \ldots k$ is an index labeling the $k$ distinct ground states. The ground states $\Psi_{0}^{a}(s)$ are not required to be exactly degenerate: they instead each have energy $E_{a}(s)$ and we denote the splitting in energy between the highest and lowest energy ground state by $\epsilon$. If there is a degeneracy of the ground states at some value of $s$, then there is some freedom in choosing the ground state basis $\Psi_{0}^{a}$; we resolve this freedom by requiring that if $E_{a}(s)=E_{b}(s)$ for some $s$, then

$$
\left\langle\Psi_{0}^{b}(s), \partial_{s} \Psi_{0}^{a}(s)\right\rangle=0 \quad \text { whenever } \quad E_{a}(s)=E_{b}(s)
$$

where $\langle\ldots, \ldots\rangle$ is used to denote the inner product. Similarly, we require that

$$
\left\langle\Psi_{0}^{a}(s), \partial_{s} \Psi_{0}^{a}(s)\right\rangle=0
$$

for all $a, s$. We label other states of the system by $\Psi^{i}, i>k$, with energy $E_{i}(s)$.

The most important result of [2] shows that the evolution of the ground state under a change in $s$ can be well approximated by a local Hermitian operator acting on the ground state if there is a gap. Let there be a gap $\Delta E$ between the sector of ground states and the rest of the spectrum for all $s$. Let $\Psi_{0}^{a}\left(s, s_{Z}\right)$ denote the set of ground states of the Hamiltonian $\mathcal{H}(s)+\left(h_{Z}\left(s_{Z}\right)-h_{Z}(s)\right)$. Then it was shown that, for any length $l_{Z}$, there exists a Hermitian operator $\mathcal{D}_{Z}^{l o c}(s)$ and an anti-Hermitian matrix $N_{b a}^{Z, l o c}$ such that $D_{Z}^{l o c}(s)$ has support on the set of sites $j$ with $\operatorname{dist}(Z, j) \leq l_{Z}$ and satisfies $\left\|\mathcal{D}_{Z}^{\text {loc }}(s)\right\| \leq t_{q}\left\|\partial_{s_{Z}} h_{Z}\left(s_{Z}\right)\right\|$, and such that $\left|\partial_{s_{Z}} \Psi_{0}^{a}\left(s, s_{Z}\right)-i \mathcal{D}_{Z}^{l o c}(s) \Psi_{0}^{a}-\sum_{b} N_{b a}^{Z, l o c}(s) \Psi_{0}^{b}(s)\right| \leq C_{1}\left(\exp \left[-l_{Z} / \xi^{\prime}\right]+|Z| \exp \left[-\mu^{\prime} l_{Z}\right]\right) t_{q}\left\|\partial_{s_{Z}} h_{Z}\left(s_{Z}\right)\right\|$,

where $C_{1}$ is some numerical constant of order unity (throughout, we denote such numerical constants by symbols $C_{1}, C_{2}, \ldots$ ) and we define the constants $\xi^{\prime}, t_{q}$ by (the $q$ in $t_{q}$ stands for "quasi-adiabatic"):

$$
\begin{gathered}
\xi^{\prime}=2 v^{\prime} / \Delta E, \\
t_{q}=\sqrt{l_{Z} / v^{\prime} \Delta E}
\end{gathered}
$$

In Eq. (17) and throughout this section, all derivatives with respect to $s_{Z}$. are taken at $s_{Z}=s$. 
From this, we can define the operator $\mathcal{D}^{l o c}(s)=\sum_{Z} \mathcal{D}_{Z}^{\text {loc }}(s)$ and bounds on $\mid \partial_{s} \Psi_{0}^{a}(s)-$ $i \mathcal{D}^{l o c}(s) \Psi_{0}^{a}-\sum_{b} N_{b a}^{l o c}(s) \Psi_{0}^{b}(s) \mid$ follow from Eq. (7), where $N_{b a}^{l o c}(s)=\sum_{Z} N_{b a}^{Z, l o c}(s)$.

We briefly sketch the derivation of Eq. (17) following ideas from [2]. We define the operator

$$
\mathcal{D}_{Z}(s)=i \int_{0}^{\infty} \mathrm{d} \tau e^{-\left(\tau / t_{q}\right)^{2} / 2}\left[\tilde{w}_{Z, s_{Z}}^{+}(i \tau)-\text { h.c. }\right]
$$

where $w_{Z, s_{Z}}=\left(\partial_{s_{Z}} h_{Z}\left(s_{Z}\right)\right)$ and where for any operator $A$ we define, following [4]:

$$
\begin{aligned}
\tilde{A}(t) & \equiv A(t) \exp \left[-\left(t / t_{q}\right)^{2} / 2\right] \\
\tilde{A}^{ \pm}(i \tau) & =\frac{1}{2 \pi} \int \mathrm{d} t \tilde{A}(t) \frac{1}{ \pm i t+\tau} .
\end{aligned}
$$

The time evolution of operators in Eq. (11) is the usual Heisenberg evolution with the Hamiltonian $\mathcal{H}(s)$. Define

$$
N_{b a}^{Z}(s)=\left\langle\Psi_{0}^{b}(s), \partial_{s_{Z}} \Psi_{0}^{a}\left(s, s_{Z}\right)\right\rangle-\left\langle\Psi_{0}^{b}(s), i \mathcal{D}_{Z}(s) \Psi_{0}^{a}(s)\right\rangle
$$

Then, one may show using the gap that for any $\Psi_{0}^{a}$ we have

$$
\begin{aligned}
& \left|\partial_{s_{Z}} \Psi_{0}^{a}\left(s, s_{Z}\right)-i \mathcal{D}_{Z}(s) \Psi_{0}^{a}(s)-\sum_{b} N_{b a}^{Z}(s) \Psi_{0}^{b}\right| \\
= & \left|-\sum_{i \neq a} \frac{1}{E_{i}(s)-E_{a}(s)}\left(\left\langle\Psi^{i}, \partial_{s_{Z}} h_{Z}\left(s_{Z}\right) \Psi_{0}^{a}\right\rangle\right) \Psi^{i}-i \mathcal{D}_{Z}(s) \Psi_{0}^{a}\left(s, s_{Z}\right)-\sum_{b} N_{b a}^{Z}(s) \Psi_{0}^{b}(s)\right| \\
\leq & \left.C_{2}\left(t_{q} \exp \left[-\left(t_{q} \Delta E\right)^{2} / 2\right]\right)\left\|\partial_{s_{Z}} h_{Z}\left(s_{Z}\right)\right\|\right) .
\end{aligned}
$$

We define $\mathcal{D}_{Z}^{\text {loc }}(s)$ as follows: define $U_{l}$ to be a unitary operator on the set of sites $j$ such that $\operatorname{dist}(Z, j)>l$. Let $\mu\left(U_{l}\right)$ be the Haar measure on such operators. Define [14]

$$
\mathcal{D}_{Z}^{l o c}(s)=\int \mathrm{d} \mu\left(U_{l_{Z}}\right) U_{l_{Z}}^{\dagger} \mathcal{D}_{Z}(s) U_{l_{Z}}
$$

Using Lieb-Robinson bounds, it is possible to show that

$$
\left\|\mathcal{D}_{Z}^{\text {loc }}(s)-\mathcal{D}_{Z}(s)\right\| \leq C_{3}\left(\exp \left[-\left(l_{Z} / v^{\prime} t_{q}\right)^{2} / 2\right]+|Z| \exp \left[-\mu^{\prime} l_{Z}\right]\right) t_{q}\left\|\partial_{s_{Z}} h_{Z}\left(s_{Z}\right)\right\|
$$

Combining these results (14]16) gives Eq. (77), where $N_{b a}^{Z, l o c}(s)=\left\langle\Psi_{0}^{b}(s), \partial_{s_{Z}} \Psi_{0}^{a}\left(s, s_{Z}\right)\right\rangle-$ $\left\langle\Psi_{0}^{b}(s), i \mathcal{D}_{Z, l o c}(s) \Psi_{0}^{a}(s)\right\rangle$. Define the operator $\left.N^{Z, l o c}(s)=\sum_{b a} N_{b a}^{Z, l o c}(s) \Psi_{0}^{b}\right\rangle\left\langle\Psi_{0}^{a}\right.$ and $N^{l o c}(s)=$ $\left.\sum_{b a} N_{b a}^{l o c}(s) \Psi_{0}^{b}\right\rangle\left\langle\Psi_{0}^{a}\right.$. 


\section{B. Berry Phase}

The discussion that follows in this subsection is meant to introduce the idea of computing a Berry phase for quasi-adiabatic evolution and show that it is close to the usual definition of the Berry phase. This discussion is not used in the rest of the paper and may be skipped if desired, but seems to be interesting in itself as well as for other future applications.

Define $P_{0}(s)$ to be the projector onto the ground state sector: $\left.P_{0}(s) \equiv \sum_{a=1}^{k} \Psi_{0}^{a}(s)\right\rangle\left\langle\Psi_{0}^{a}(s)\right.$. A direct computation shows that for all $a, b$

$$
\left|\left\langle\Psi_{0}^{b}(s), \mathcal{D}_{Z, l o c}(s) \Psi_{0}^{a}(s)\right\rangle\right| \leq C_{4}\left(t_{q} \exp \left[-l_{Z} / \xi^{\prime}\right]+t_{q}|Z| \exp \left[-\mu^{\prime} l_{Z}\right]+t_{q}^{2} \epsilon\right) s\left\|\partial_{s_{Z}} h\left(s_{Z}\right)\right\|
$$

Note that for large $l_{Z}$ and small $\epsilon$, the right-hand side of this equation becomes close to zero, giving a result similar to Eqs. (5),6); this will be used to relate the evolution in the ground state sector produced by $i \mathcal{D}(s)$ to a Berry phase.

Eq. (7) gives the evolution of wavefunctions under an infinitesimal change in $s$. We now present a unitary operator $V$ to describe the change in wavefunctions from $s=0$ to $s=1$. We define

$$
V(s) \equiv \mathcal{S}^{\prime} \exp \left[\int_{0}^{s} \mathrm{~d} s^{\prime} i \mathcal{D}\left(s^{\prime}\right)\right]
$$

where $\mathcal{S}^{\prime}$ denotes that the exponential is $s^{\prime}$-ordered. Then, $V \Psi_{0}^{a}(0)$ is close in norm to some linear combination of states $\Psi_{0}^{b}(s)$, with an error that may be estimated from Eq. (77). If the Hamiltonian is quasi-adiabatically continued around a closed path in parameter space, so that $\mathcal{H}\left(s_{\text {final }}\right)=\mathcal{H}(0)$, then $\left|V\left(s_{\text {final }}\right) \Psi_{0}^{a}-\sum_{b} Q_{b a} \Psi_{0}^{b}\right| \leq C_{1} \sum_{Z}\left(\exp \left[-l_{Z} / \xi^{\prime}\right]+\right.$ $\left.|Z| \exp \left[-\mu^{\prime} l_{Z}\right]\right) t_{q} s_{\text {final }}\left\|\partial_{s_{Z}} h\left(s_{Z}\right)\right\|$ where

$$
\begin{aligned}
Q & =\mathcal{S}^{\prime} \exp \left[\int_{0}^{s} \mathrm{~d} s^{\prime} i P_{0}\left(s^{\prime}\right) \mathcal{D}\left(s^{\prime}\right) P_{0}\left(s^{\prime}\right)-P_{0} \sum_{a}\left(\partial_{s^{\prime}} \Psi_{0}^{a}\left(s^{\prime}\right)\right)\right\rangle\left\langle\Psi_{0}^{a}\left(s^{\prime}\right)\right] \\
& =\mathcal{S}^{\prime} \exp \left[-\int_{0}^{s} \mathrm{~d} s^{\prime} N^{l o c}\left(s^{\prime}\right)\right] .
\end{aligned}
$$

Eq. (17) gives

$$
\left\|Q-Q^{b e r r y}\right\| \leq C_{4} \sum_{Z}\left(t_{q} \exp \left[-l_{Z} / \xi^{\prime}\right]+t_{q}|Z| \exp \left[-\mu^{\prime} l_{Z}\right]+t_{q}^{2} \epsilon\right) s_{\text {final }}\left\|\partial_{s_{Z}} h\left(s_{Z}\right)\right\|,
$$

where $Q^{\text {berry }}$ is the non-Abelian Berry phase for an adiabatic evolution in a related system in which the level splitting between the ground states $\Psi_{0}^{a}(s)$ is set equal to zero[15].

While we used the assumption that there is a gap $\Delta E$ to derive Eq. (14), it is important to note that this equation in fact depends only on the weaker assumption that the state 
$\partial_{s} h_{Z}(s) \Psi_{0}^{a}$ is a linear combination of ground states and states $\Psi^{i}$ with $E_{i} \geq \Delta E+E_{a}$ for $a=1 \ldots k$. This follows automatically if there is a gap in the spectrum, but it also follows in a spin system if, for example, there is a gap $\Delta E$ between the ground state with spin-0 and the lowest spin-1 state, and $\partial_{s} h_{Z}(s)$ has spin-1.

\section{GOLDSTONE'S THEOREM IN SPIN-GAPPED SYSTEMS}

\section{A. Statement of Result and Examples}

Recently, the exponential clustering of correlation functions in a system with a gap has been proven [3, 4, 5, 6]. That is, it has been shown that, for a local Hamiltonian with a gap $\Delta E$ between a sector of degenerate ground states, labeled $\Psi_{0}^{a}$ for $a=1 \ldots k$, and the rest of the spectrum, the connected correlated function, $\langle A B\rangle-\left\langle A P_{0} B\right\rangle$, of two operators $A, B$ with support on sets $X, Y$ is exponentially small in the distance $\operatorname{dist}(X, Y)$. Here, $\langle O\rangle \equiv k^{-1} \sum_{a=1}^{k}\left\langle\Psi_{0}^{a}, O \Psi_{0}^{a}\right\rangle$ and $\left.P_{0} \equiv \sum_{a=1}^{k} \Psi_{0}^{a}\right\rangle\left\langle\Psi_{0}^{a}\right.$ is the projector on the ground state sector. For a parameter dependent Hamiltonian, we define $\langle O\rangle_{s} \equiv k^{-1} \sum_{a=1}^{k}\left\langle\Psi_{0}^{a}(s), O \Psi_{0}^{a}(s)\right\rangle$. In the event that the states $\Psi_{0}^{a}$ are not exactly degenerate but only approximately degenerate, similar bounds were found with corrections that depend on the energy difference between the states.

In this section we prove a stronger statement about the decay of correlation functions in gapped systems with a continuous symmetry. We consider Hamiltonians which obey Eq. (1) for some $\mu, s_{1}$ and we also require that for any site $i$ the number of sites $j$ within distance $l$ of site $i$ is bounded by $a l^{d}$ for some finite $a, d$ :

$$
\sum_{j, \operatorname{dist}(i, j) \leq l} 1 \leq a l^{d}
$$

We consider Hamiltonians which have a $U(1)$ symmetry as follows: for each site $i$ we assume that there exists a local operator $q_{i}$ with support on that site such that $[Q, \mathcal{H}]=0$ where $Q=\sum_{i} q_{i}$. For any set $X$, we define $R(\theta, X)=\prod_{i \in X} \exp \left[i q_{i} \theta\right]$. We consider operators $\phi_{X}, \bar{\phi}_{Y}$ with support on sets $X, Y$ which transform as vectors as follows under this $U(1)$ symmetry: $R(-\theta, X) \phi_{X} R(\theta, X)=\exp [i \theta] \phi_{X}$ and $R(-\theta, Y) \bar{\phi}_{Y} R(\theta, Y)=\exp [-i \theta] \bar{\phi}_{Y}$. We assume that $\left\|q_{i}\right\| \leq q_{\max }$ for some $q_{\max }$. We will show that, for a Hamiltonian which obeys all these 
conditions and has a gap $\Delta E$ between the ground state sector and the rest of the spectrum

$$
\begin{aligned}
\left\langle\phi_{X} \bar{\phi}_{Y}\right\rangle \leq & C_{5}\left\|\phi_{X} \bar{\phi}_{Y}\right\||X| a \operatorname{dist}(X, Y)^{d} s_{1} q_{\max } \sqrt{\operatorname{dist}(X, Y) \Delta E / v^{\prime}} \times \\
& \left(\exp \left[-\mu^{\prime} \operatorname{dist}(X, Y) / 2\right]+\exp \left[-\operatorname{dist}(X, Y) / 2 \xi^{\prime}\right]\right)
\end{aligned}
$$

Picking the optimal value of $\mu^{\prime}$ in Eq. (41), we show that

$$
\left\langle\phi_{X} \bar{\phi}_{Y}\right\rangle \leq C_{6}\left\|\phi_{X} \bar{\phi}_{Y}\right\||X| \operatorname{dist}(X, Y)^{d} q_{\max } s_{1} \sqrt{\operatorname{dist}(X, Y) \Delta E / v^{\prime}} \exp [-\operatorname{dist}(X, Y) / 2 \xi]
$$

with

$$
\xi=2 v / \Delta E+\mu
$$

We emphasize that we do not require the states in the ground state sector to be degenerate with each other, simply the existence of a gap between that sector and the rest of the spectrum. Further, the bounds do not depend on the value of $k$ or on energy difference between the states $\Psi_{0}^{a}$. This result (22,23) is stronger than that in [5, 16] which was only valid for $d<2$ while the present result is valid in arbitrary dimension. It is also stronger than other previous results[17] which either required a unique ground state or else assumed an ergodic property which is equivalent to requiring the vanishing of the matrix elements in the ground state sector; in either of these cases the decay of correlations becomes equivalent to clustering.

Before giving the proof we motivate the definition of $q_{i}$ by discussing physical examples. In a Bose system with conserved particle number, the $q_{i}$ can represent the particle number on a given site and the operators $\phi_{X}, \bar{\phi}_{Y}$ can represent creation and annihilation operators for the bosons. For a spin system, the $q_{i}$ can represent the $z$ component of the spin on a site and the $\phi_{X}, \bar{\phi}_{Y}$ can represent raising and lowering spin operators on sites. Consider such a spin system in a disordered quantum paramagnetic phase. An example of such a system would be obtained by a two dimensional Hamiltonian of spin- $1 / 2$ spins for a system of two layers with coordinates labeled $(i, l)$ where $l=1,2$ is a layer label and $i$ indexes position in each layer. First consider the Hamiltonian:

$$
\mathcal{H}=J \sum_{i, j n . n .} \vec{S}_{i, 1} \cdot \vec{S}_{j, 1}+J_{\perp} \sum_{i} \vec{S}_{i, 1} \cdot \vec{S}_{i, 2}
$$

with $J, J_{\perp}>0$ where the first sum is over nearest neighbor $i, j$. This is a bilayer model Heisenberg model that is connected with the Kondo lattice model[18]. For $J_{\perp}>>J$, the 

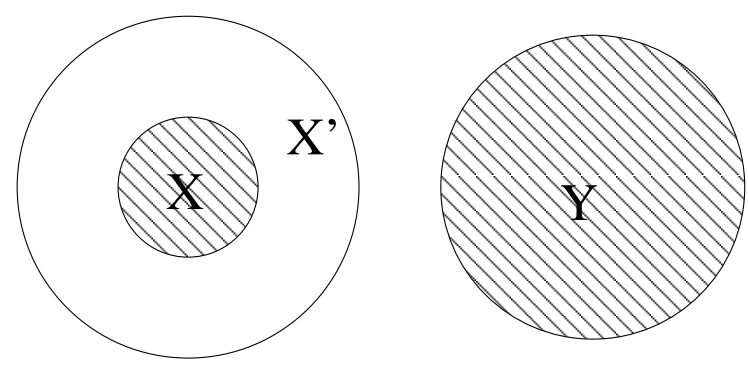

FIG. 1: Illustration of the geometry we consider. $X, Y$ are shown as shaded regions, while $X^{\prime}$ includes everything within the outer circle around $X$.

ground state is unique and gapped, with spins $(i, 1),(i, 2)$ being in a singlet state with high probability. In this case, Eq. (22) shows $\left\langle S_{i, 1}^{x} S_{j, 1}^{x}\right\rangle$ is exponentially decaying in $\operatorname{dist}(i, j)$ but this provides no additional information beyond that already known from the exponential decay of correlation functions. Now consider the Hamiltonian on a lattice with two "defects": for some given $k, l$, we replace the spins at sites $(k, 2)$ and $(l, 2)$ with spin-0 spins[19]. That is, we remove them from the lattice, so $\vec{S}_{k, 2}=\vec{S}_{l, 2}=0$. Then, there are unpaired spins at $(k, 1)$ and $(l, 1)$. In the limit $J=0$, indeed there are four exactly degenerate ground states. Returning to the case $J_{\perp}>>>0$, we expect that if $\operatorname{dist}(k, l)$ is large then there will be four low energy states and then a gap to the rest of the spectrum. In this case, Eq. (22) can be used, given the assumption of a gap as all other requirements are trivially satisfied, to bound $\left\langle S_{k, 1}^{x} S_{l, 1}^{x}\right\rangle$ and hence while the operators $\vec{S}_{k, 1}, \vec{S}_{l, 1}$ may have nonvanishing matrix elements between these states, the average of the correlation function over the different ground states is small

\section{B. Proof}

To show Eq. (22), we define a set of parameter dependent Hamiltonians $\mathcal{H}_{\theta}=\sum_{Z} h_{Z}(\theta)$ as follows. Let $X^{\prime}$ denote the set of sites $i$ such that $\operatorname{dist}(X, i) \leq \operatorname{dist}(X, Y) / 2$, as shown in the figure. Then define $h_{Z}(\theta)=R\left(X^{\prime}, \theta\right) h_{Z} R\left(X^{\prime},-\theta\right)$. Clearly, as $R\left(X^{\prime},-\theta\right)$ is a unitary transformation, $\mathcal{H}_{\theta}$ has the same spectrum of $\mathcal{H}$ and the ground states of $\mathcal{H}_{\theta}$ are given by $\Psi_{0}^{a}(\theta)=R\left(X^{\prime}, \theta\right) \Psi_{0}(\theta)$.

Thus,

$$
\partial_{\theta}\left\langle\phi_{X} \bar{\phi}_{Y}\right\rangle_{\theta}=\partial_{\theta}\left\langle R\left(X^{\prime},-\theta\right) \phi_{X} \bar{\phi}_{Y} R\left(X^{\prime}, \theta\right)\right\rangle
$$




$$
=\partial_{\theta} \exp [i \theta]\left\langle\phi_{X} \bar{\phi}_{Y}\right\rangle=i\left\langle\phi_{X} \bar{\phi}_{Y}\right\rangle
$$

where we used the fact that $X \subset X^{\prime}$ while $Y \cap X^{\prime}=0$ so that $\left[\bar{\phi}_{Y}, R\left(X^{\prime}, \theta\right)\right]=0$ and where we evaluate the derivatives at $\theta=0$.

Without loss of generality we may assume that

$$
\left[Q, h_{Z}\right]=0
$$

for all $Z$. To show this, we use $[Q, \mathcal{H}]=0$ to write $\mathcal{H}=\sum_{Z} O_{Z}$ where $O_{Z} \equiv$ $(1 / 2 \pi) \int_{0}^{2 \pi} \mathrm{d} \theta \exp [i Q \theta] h_{Z} \exp [-i Q \theta]$. Then, $O_{Z}$ has support on $Z$ and $\left\|O_{Z}\right\| \leq\left\|h_{Z}\right\|$. Finally, $\left[Q, O_{Z}\right]=0$. Hence, by replacing $h_{Z}$ by the operators $O_{Z}$, we succeed in rewriting the Hamiltonian such that Eq. (27) is satisfied and such that the Hamiltonian still obeys Eq. (11). From Eq. (27),

$$
\begin{aligned}
& Z \cap X^{\prime}=0 \rightarrow h_{Z}(\theta)=h_{Z}, \\
& Z \subseteq X^{\prime} \rightarrow h_{Z}(\theta)=h_{Z} .
\end{aligned}
$$

That is, $h_{Z}(\theta)=h_{Z}$ unless $Z$ contains some point $i \in X^{\prime}$ and some other point $j \notin X^{\prime}$.

We now use quasi-adiabatic continuation to bound the first line of Eq. (26), thus bounding the correlation function on the last line of this equation. Using $\partial_{\theta}\left\langle\phi_{X} \bar{\phi}_{Y}\right\rangle_{\theta}=$ $\frac{1}{k} \sum_{a=1}^{k} \partial_{\theta}\left\langle\Psi_{0}^{a}(\theta), \phi_{X} \bar{\phi}_{Y} \Psi_{0}^{a}(\theta)\right\rangle$ and Eq. (7) we have

$$
\begin{aligned}
\left|\partial_{\theta}\left\langle\phi_{X} \bar{\phi}_{Y}\right\rangle_{\theta}\right| \leq & \mid \sum_{Z} \frac{1}{k} \sum_{a=1}^{k}\left(i\left\langle\Psi_{0}^{a}(\theta),\left[\phi_{X} \bar{\phi}_{Y}, \mathcal{D}_{Z}^{l o c}(\theta=0)\right] \Psi_{0}^{a}(\theta)\right\rangle+\right. \\
& \left.\sum_{b=1}^{k} N_{b a}^{Z, l o c}(\theta=0)\left\langle\Psi_{0}^{a}(\theta), \phi_{X} \bar{\phi}_{Y} \Psi_{0}^{b}(\theta)\right\rangle+\sum_{b=1}^{k} N_{b a}^{Z, l o c}(\theta=0)^{*}\left\langle\Psi_{0}^{b}(\theta), \phi_{X} \bar{\phi}_{Y} \Psi_{0}^{a}(\theta)\right\rangle\right) \mid \\
& +C_{1} \sum_{Z}\left(\exp \left[-l_{Z} / \xi^{\prime}\right]+|Z| \exp \left[-\mu^{\prime} l_{Z}\right]\right) t_{q}\left\|\partial_{\theta_{Z}} h_{Z}\left(\theta_{Z}\right)\right\|\left\|\phi_{X} \bar{\phi}_{Y}\right\|
\end{aligned}
$$

The terms involving the matrix $N^{Z, l o c}$ in Eq. (29) cancel after summing over $a$ and $b$. This is the key step which makes the quasi-adiabatic continuation useful in deriving the desired exponential decay of correlations. We will now show that all the other terms on the right-hand side of Eq. (29) are exponentially small in $\operatorname{dist}(X, Y)$, when we choose $l_{Z}=\min (\operatorname{dist}(X, Z), \operatorname{dist}(Y, Z))-1$. This requires a straightforward, but lengthy, series of inequalities.

The commutator $\left[\phi_{X} \bar{\phi}_{Y}, \mathcal{D}_{Z}^{\text {loc }}(\theta=0)\right]$ is vanishing unless $\mathcal{D}_{Z}^{\text {loc }}(\theta=0)$ has support on either set $X$ or set $Y$ which, with the above choice of $l_{Z}$, occurs only if $Z \cap X \neq \emptyset$ or $Z \cap Y \neq \emptyset$. 
Further, from Eq. (28) $\mathcal{D}_{Z}^{\text {loc }}(\theta=0)$ is vanishing unless $Z$ includes some point $i \in X^{\prime}$ and some other point $j \notin X^{\prime}$. Thus, the commutator $\left[\phi_{X} \bar{\phi}_{Y}, \mathcal{D}_{Z}^{\text {loc }}(\theta=0)\right]$ is vanishing unless either $Z$ includes some point $i \in X$ and some other point $j \notin X^{\prime}$ or else $Z$ includes some point $i \in X^{\prime}$ and some other point $j \in Y$. In either case, we have $i \in X^{\prime}$ and $\operatorname{dist}(i, j) \geq \operatorname{dist}(X, Y) / 2$. Thus,

$$
\begin{aligned}
\sum_{Z}\left\|\left[\phi_{X} \bar{\phi}_{Y}, \mathcal{D}_{Z}^{\text {loc }}(\theta=0)\right]\right\| & \leq 2 t_{q}\left\|\phi_{X} \bar{\phi}_{Y}\right\| \sum_{i \in X^{\prime}} \sum_{j, \operatorname{dist}(i, j) \geq \operatorname{dist}(X, Y) / 2} \sum_{Z \ni i, j}\left\|\partial_{\theta_{Z}} h_{Z}\left(\theta_{Z}\right)\right\| \\
& \leq 4 t_{q} q_{\text {max }}\left\|\phi_{X} \bar{\phi}_{Y}\right\|\left|X^{\prime}\right| s_{1} \exp \left[-\mu^{\prime}(\operatorname{dist}(X, Y) / 2)\right] \\
& \leq 4 t_{q} q_{\text {max }}\left\|\phi_{X} \bar{\phi}_{Y}\right\| a(\operatorname{dist}(X, Y) / 2)^{d}|X| s_{1} \exp \left[-\mu^{\prime}(\operatorname{dist}(X, Y) / 2)\right],
\end{aligned}
$$

where we used Eqs. (1421) and $\left\|\mathcal{D}_{Z}^{\text {loc }}\right\| \leq t_{q}\left\|\partial_{\theta_{Z}} h_{Z}\left(\theta_{Z}\right)\right\|$ and $\left\|\partial_{\theta_{Z}} h_{Z}\left(\theta_{Z}\right)\right\| \leq 2 q_{\max }\left\|h_{Z}\right\|$ for the last two inequalities.

Similarly, noting that $l_{Z} \geq \operatorname{dist}(X, Y) / 2-\operatorname{diam}(Z)$,

$$
\begin{aligned}
& \sum_{Z}\left|\left(\exp \left[-l_{Z} / \xi^{\prime}\right]+|Z| \exp \left[-\mu^{\prime} l_{Z}\right]\right)\right| t_{q}\left\|\partial_{\theta_{Z}} h_{Z}\left(\theta_{Z}\right)\right\| \\
\leq & \sum_{i \in X^{\prime}} \sum_{Z \ni i} 2\left(\exp \left[-(\operatorname{dist}(X, Y) / 2-\operatorname{diam}(Z)) / \xi^{\prime}\right]+|Z| \exp \left[-\mu^{\prime}(\operatorname{dist}(X, Y) / 2-\operatorname{diam}(Z)]\right) t_{q} q_{\text {max }}\left\|h_{Z}\right\|\right. \\
\leq & |X| a(\operatorname{dist}(X, Y) / 2)^{d} s_{1} q_{\text {max }} \sqrt{\operatorname{dist}(X, Y) \Delta E / v^{\prime}}\left(\exp \left[-\mu^{\prime} \operatorname{dist}(X, Y) / 2\right]+\exp \left[-\operatorname{dist}(X, Y) / 2 \xi^{\prime}\right]\right) .
\end{aligned}
$$

Combining Eqs.(30),31), we find that

$$
\begin{aligned}
\left|\partial_{\theta}\left\langle\phi_{X} \bar{\phi}_{Y}\right\rangle_{\theta}\right| \leq & C_{5}\left\|\phi_{X} \bar{\phi}_{Y}\right\||X| a \operatorname{dist}(X, Y)^{d} s_{1} q_{\max } \sqrt{\operatorname{dist}(X, Y) \Delta E / v^{\prime}} \times \\
& \left(\exp \left[-\mu^{\prime} \operatorname{dist}(X, Y) / 2\right]+\exp \left[-\operatorname{dist}(X, Y) / 2 \xi^{\prime}\right]\right) .
\end{aligned}
$$

Combining this with Eq. (26), we arrive at Eq.(22), as desired.

It is interesting to note that Eq. (21) is necessary in this derivation. We sketch a system for which Eq. (21) does not hold, and show how a Goldstone theorem may fail in this case. Consider a random graph with $V$ nodes each having coordination number 3 . Consider a set of $V$ spin- $1 / 2$ spins, with Hamiltonian

$$
H=-\sum_{i, j} J_{i j} \vec{S}_{i} \cdot \vec{S}_{j}
$$

where the interaction matrix $J_{i j}$ equals 1 if the nodes $i, j$ are connected by an edge on the graph, and zero otherwise. The interaction is ferromagnetic, so pointing all spins up (or in any other direction) gives a ground state. Further, the Hamiltonian is local, using a 
shortest path metric on the graph to define dist $(i, j)$. However, a random graph of this form is typically an expander graph[20] with a gap in the spectrum of the graph Laplacian, so a spin-wave theory calculation[21] gives a gap in the magnon spectrum. Thus, this system has a set of degenerate ground states and a gap. However, the spin correlations do not decay, as $\left\langle\vec{S}_{i} \cdot \vec{S}_{j}\right\rangle=1 / 4$ for all $i \neq j$.

\section{FLUX QUANTIZATION IN SPIN-GAPPED SYSTEMS}

\section{A. Examples and Statement of Result}

In this section we consider flux periodicity in systems of fermions with half-integer spin. Before stating the precise result we motivate some of the definitions through physical examples. Consider a system of fermions on a cylinder, and suppose that an additional magnetic field is applied through the cylinder, introducing an Aharonov-Bohm phase for the fermions. The Hamiltonian is unchanged, up to a gauge transformation, if the magnetic flux through the cylinder is changed by $2 \pi$, and hence the ground state energy is periodic in the flux with periodic $2 \pi$. For an arbitrary Hamiltonian the energy of the ground state for $\pi$ flux may be much different for that at 0 flux. However, a superconductor typically has its ground state energy approximately periodic in the flux with period $\pi$ due to so-called Byer-Yang states[8], up to some corrections which are exponentially small in system size $L$. This is often taken to imply that the elementary excitation in the system has charge $2 e$ rather than $e$.

However, there are other interesting scenarios in which the ground state energy at $\pi$ flux is close to that at zero flux. Consider a system at half filling which forms a resonating valence bond state (RVB) with a spin gap. Then dope this system by introducing a small number of holes. The holes have vanishing spin and charge $+e$, and may Bose condense, again giving a superconductor. Naively, one may expect that in this case the ground state energy of the system at $\pi$ flux would be very different from that at zero flux as the excitations that condense have charge $e$. However, the presence of topological excitations of the valence bond system implies that in fact the ground state energy is still approximately periodic in the flux with period $\pi[9,22,23]$. The question was raised in [9] whether there were some general conditions under which one could prove that this approximate periodicity held for fermionic system. In this section, we provide a partial answer to this question by considering the case 
in which the system has a spin gap, as would occur for an $s$-wave superconductor or for a short wave RVB state, and prove that there exists a state of the system at $\pi$ flux which is close to the ground state energy at zero flux. Unfortunately, our results will not apply to $d$-wave superconductors where there are gapless spin excitations.

We now state the result. We assume that there are conserved spin and charge as follows. On each site $i$ we assume that there is a Hilbert with dimension 4, representing the states of no fermions, one fermion with spin up, one fermion with spin down, and two fermions. We define the spin operator on site $i, S_{i}^{z}$, to be diagonal in this basis with eigenvalues $0,+1 / 2,-1 / 2,0$ respectively, and we define the fermion number operator on site $i, q_{i}$, to be diagonal in this basis with eigenvalues $0,1,1,2$. We assume that the Hamiltonian $\mathcal{H}$ commutes with the operators $S^{z}=\sum_{i} S_{i}^{z}$ and $Q=\sum_{i} q_{i}$; as before, this means that we may assume without loss of generality that $\left[S^{z}, h_{Z}\right]=\left[Q, h_{Z}\right]=0$ for all $Z$. Note that $S_{i}^{z} \pm q_{i} / 2$ has integer eigenvalues. We assume that the Hamiltonian is local in the sense of Eq. (11) and we assume that the system is periodic in one direction as follows: let the system be defined on a $d$ dimensional lattice of $V$ different sites, with any site $i$ having coordinates $\left(x_{0}(i), x_{1}(i), \ldots x_{d-1}(i)\right)$, with periodic boundary conditions in the $x_{0}$ direction with period $L$. Assume that $\operatorname{dist}(i, j) \geq \min _{n}\left|x_{0}(i)-x_{0}(j)-n L\right|$, where the minimum is over all integers $n$. This is satisfied by any physically reasonable metric on the lattice, such as a Manhattan metric.

We assume that $\mathcal{H}$ has a unique ground state $\Psi_{0}$, although the results can be readily extended to a system with multiple ground states with small splitting $\epsilon$. We define a Hamiltonian $\mathcal{H}$ to have a spin gap $\Delta E$ if the the ground state has vanishing spin and the first excited state with non-vanishing spin has energy at least $\Delta E$ above the ground state energy.

We now define the Hamiltonians $\mathcal{H}_{\theta}$ with flux $\theta$ inserted. Define $\mathcal{L}$ to be the set of all points $i$ with $0 \leq x_{0}(i)<L / 2$. For any set $X$, define $R_{q}(\theta, X)=\prod_{i \in X} \exp \left[i q_{i} \theta\right]$. Define $\mathcal{H}_{\theta}=\sum_{Z} h_{Z}(\theta)$, where we define $h_{Z}$ as follows: if $Z$ contains any points $i$ with $L / 4 \leq x_{0}(i) \leq 3 L / 4$, then we define

$$
h_{Z}(\theta)=R_{q}(-\theta) h_{Z} R_{q}(\theta)
$$

Otherwise, we define

$$
h_{Z}(\theta)=h_{Z}
$$

We will prove that, under the conditions identified above, (conserved spin and charge, 


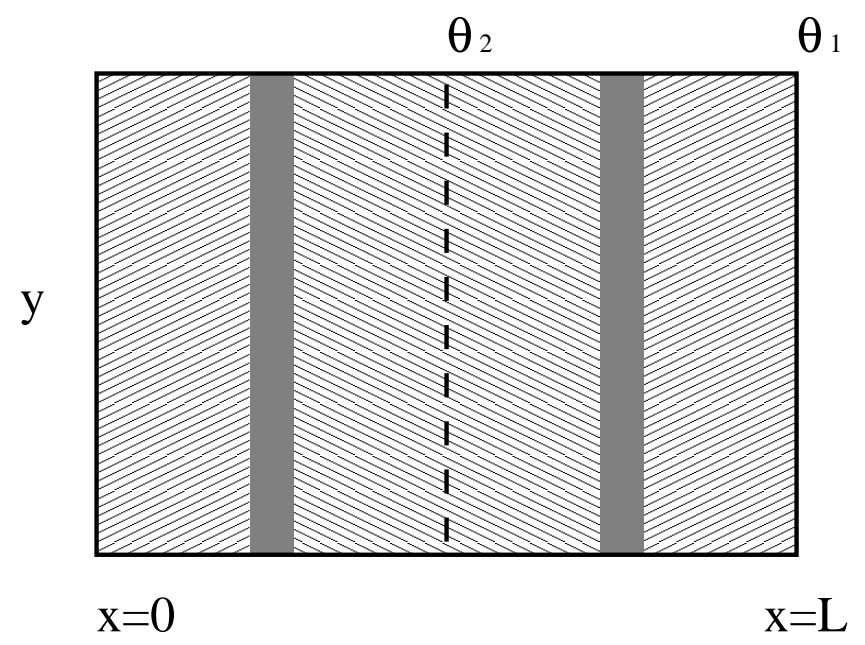

FIG. 2: Illustration of the geometry we consider. Flux is inserted along the dashed line in the center, and at the edge. The slanting upwards line is the support of $W_{1}$, the slanting downwards is the support of $W_{2}$. The grey areas have width $L / 2(\mu \xi+1)$. The lines $x_{0}(i)=L / 4$ and $x_{0}(i)=3 L / 4$ pass vertically through the middle of the left and right grey areas, respectively.

half-integer spin, locality, periodicity, uniqueness of ground state, and spin gap), that there exists some state, which we will write as $W_{1}(\pi) \Psi_{0}$ with $W_{1}$ a unitary operator, such that the difference in energies

$$
\left|\left\langle W_{1}(\pi) \Psi_{0}, \mathcal{H}_{\pi} W_{1}(\pi) \Psi_{0}\right\rangle-\left\langle\Psi_{0}, \mathcal{H} \Psi_{0}\right\rangle\right| \leq C_{7} V^{2} \sqrt{L / v \Delta E} s_{1}^{2} \exp [-L / 4(\xi+1 / \mu)]
$$

Note that $W_{1}(\pi) \Psi_{0}$ need not be the ground state of $\mathcal{H}_{\pi}$.

\section{B. Proof}

In this subsection we provide the proof. The main idea is to define separate fluxes for up and down fermions separate, and use quasi-adiabatic continuation to carry one flux from 0 to $\pi$ and the other from 0 to $-\pi$. Then, the proof closely follows the proof of a higher dimensional Lieb-Schultz-Mattis system given in[24]. For any set $X$, define $R_{\uparrow}(\theta, X)=$ $\prod_{i \in X} \exp \left[i\left(S_{i}^{z}+q_{i} / 2\right) \theta\right]$ and $R_{\downarrow}(\theta, X)=\prod_{i \in X} \exp \left[i\left(-S_{i}^{z}+q_{i} / 2\right) \theta\right]$. Thus, $R_{\uparrow}$ produces a gauge transformation on the up spin fermions and $R_{\downarrow}$ transforms the down spin fermions. Note that $R_{\uparrow}(\pi, X) R_{\downarrow}(-\pi, X)=R_{q}(\pi, X)$; this equality depends on the fact that $R_{\downarrow}(-\pi, X)=$ $R_{\downarrow}(\pi, X)$. 
We now define a family of Hamiltonian with separate up and down fluxes inserted, and we also insert flux at two different points: along a line with $x_{0}=0$ and along a line with $x_{0}=L / 2$ as shown in the figure. By gauge invariance the spectrum of the Hamiltonian depends only on the total of these fluxes, but the introduction of two different flux angles is a useful technical device. We define four flux angles, $\theta_{1}^{\uparrow}, \theta_{1}^{\downarrow}, \theta_{2}^{\uparrow}, \theta_{2}^{\downarrow}$, where the fluxes described by angles $\theta_{1}$ are along the line with $x_{0}=0$ and the fluxes described by angles $\theta_{2}$ are along the line with $x_{0}=L / 2$, and where we introduce different fluxes for up and down particles. The insertion of physical magnetic flux into into the system the system leads to $\theta_{1}^{\downarrow}=\theta_{1}^{\uparrow}$ and $\theta_{2}^{\downarrow}=\theta_{2}^{\uparrow}$, however our introduction of separate angles for up and down particles is necessary to derive the final result. The resulting Hamiltonian $\mathcal{H}\left(\theta_{1}^{\uparrow}, \theta_{1}^{\downarrow}, \theta_{2}^{\uparrow}, \theta_{2}^{\downarrow}\right)=\sum_{Z} h_{Z}\left(\theta_{1}^{\uparrow}, \theta_{1}^{\downarrow}, \theta_{2}^{\uparrow}, \theta_{2}^{\downarrow}\right)$, where we define $h_{Z}$ as follows: if $Z$ contains any points $i$ with $L / 4 \leq x_{0}(i) \leq 3 L / 4$, then we define

$$
h_{Z}\left(\theta_{1}^{\uparrow}, \theta_{1}^{\downarrow}, \theta_{2}^{\uparrow}, \theta_{2}^{\downarrow}\right)=R_{\uparrow}\left(-\theta_{2}^{\uparrow}, \mathcal{L}\right) R_{\downarrow}\left(-\theta_{2}^{\downarrow}, \mathcal{L}\right) h_{Z} R_{\uparrow}\left(\theta_{2}^{\uparrow}, \mathcal{L}\right) R_{\downarrow}\left(\theta_{2}^{\downarrow}, \mathcal{L}\right) .
$$

Otherwise, we define

$$
h_{Z}\left(\theta_{1}^{\uparrow}, \theta_{1}^{\downarrow}, \theta_{2}^{\uparrow}, \theta_{2}^{\downarrow}\right)=R_{\uparrow}\left(\theta_{1}^{\uparrow}, \mathcal{L}\right) R_{\downarrow}\left(\theta_{1}^{\downarrow}, \mathcal{L}\right) h_{Z} R_{\uparrow}\left(-\theta_{1}^{\uparrow}, \mathcal{L}\right) R_{\downarrow}\left(-\theta_{1}^{\downarrow}, \mathcal{L}\right) .
$$

Then, if $\theta_{1}^{\uparrow}=-\theta_{2}^{\uparrow}$ and $\theta_{1}^{\downarrow}=-\theta_{2}^{\downarrow}$, we have $\Psi_{0}\left(\theta_{1}^{\uparrow}, \theta_{1}^{\downarrow},-\theta_{1}^{\uparrow},-\theta_{1}^{\downarrow}\right)=R_{\uparrow}\left(\theta_{1}^{\uparrow}, \mathcal{L}\right) R_{\downarrow}\left(\theta_{1}^{\downarrow}, \mathcal{L}\right) \Psi_{0}$. Further, the eigenvalues of the Hamiltonian $\mathcal{H}\left(\theta_{1}^{\uparrow}, \theta_{1}^{\downarrow}, \theta_{2}^{\uparrow}, \theta_{2}^{\downarrow}\right)$ depend only on $\theta_{1}^{\uparrow}+\theta_{2}^{\uparrow}$ and $\theta_{1}^{\downarrow}+\theta_{2}^{\downarrow}$. We define $\mathcal{Z}_{c}$ to be the set of $Z$ such that $h_{Z}\left(\theta_{1}^{\uparrow}, \theta_{1}^{\downarrow}, \theta_{2}^{\uparrow}, \theta_{2}^{\downarrow}\right)$ is different from $h_{Z}$. Crucially, the physical Hamiltonian with $\pi$ magnetic flux inserted is

$$
\mathcal{H}_{\pi}=\mathcal{H}(\pi, \pi, 0,0)
$$

The proof now closely follows[24], and we only sketch the proof; the only differences from [24] are the presence of separate flux angles for up and down fermions, and some minor complications due to exponentially decaying interactions, rather than finite range. We define $W_{1}(\phi)$ to be the operator which quasi-adiabatically continues $\mathcal{H}(\theta,-\theta, 0,0)$ from $\theta=0$ to $\theta=\phi$, using Eq. (18) with $l_{Z}=L / 4-\operatorname{diam}(Z)-L / 4(\xi \mu+1)$. We define $W_{2}(\phi)$ to be the operator which quasi-adiabatically continues $\mathcal{H}(0,0, \theta,-\theta)$ from $\theta=0$ to $\theta=-\phi$. We define $W(\phi)$ to be the operator which quasi-adiabatically continues $\mathcal{H}(\theta,-\theta,-\theta, \theta)$ from $\theta=0$ to $\theta=\phi$.

Following the results in [24] one may show that

$$
\left|R_{\uparrow}(\pi, X) R_{\downarrow}(-\pi, X) \Psi_{0}-W(\pi) \Psi_{0}\right| \leq c_{2}
$$


where

$$
\begin{aligned}
c_{2} & =C_{8} \sum_{Z, Z \in \mathcal{Z}_{c}} t_{q}\left\|h_{Z}\right\|\left(\exp \left[-l_{Z} / \Delta E / 2 v^{\prime}\right]+|Z| \exp \left[-\mu^{\prime} l_{Z}\right]\right) \\
& \leq C_{8} \sqrt{L / v \Delta E} s_{1} V\left(\exp \left[-l_{Z} / \Delta E / 2 v^{\prime}\right]+|Z| \exp \left[-\mu^{\prime} l_{Z}\right]\right)
\end{aligned}
$$

and also that

$$
\left\|W_{1}(\pi) W_{2}(\pi)-W(\pi)\right\| \leq c_{3}
$$

where

$$
c_{3}=C_{9} \sum_{Z, Z \in \mathcal{Z}_{c}} t_{q}\left\|h_{Z}\right\|\left(\exp \left[-l_{Z} / \Delta E / 2 v^{\prime}\right]+|Z| \exp \left[-\mu^{\prime} l_{Z}\right]\right)
$$

Here, we use constants $c_{2}, c_{3}$ to follow the notation of [24].

Eq. (42) relies on the fact that $W_{1}(\pi)$ and $W_{2}(\pi)$ can be written as exponentials of local operators $\mathcal{D}_{1}, \mathcal{D}_{2}$. The commutator of $\mathcal{D}_{1}$ with $\mathcal{D}_{2}$ can be bounded, and thus one can "reorder" the quasi-adiabatic evolution so that instead of first evolving with $W_{2}$ and then with $W_{1}$, both flux angles are changed at the same time, as with $W$.

Eq. (40) relies on the existence of a spin gap in the system. For a gapped system with a unique ground state, the quasi-adiabatic evolution approximately evolves the ground state of the initial Hamiltonian, $\Psi_{0}$, into the ground state of the final Hamiltonian, $R_{\uparrow}(\pi, X) R_{\downarrow}(-\pi, X) \Psi_{0}$. Here, we have not specified that the Hamiltonian has a gap. However, $\partial_{\theta} \mathcal{H}(\theta,-\theta,-\theta, \theta)$ is a spin-1 operator, and hence the spin gap suffices to show Eq. (40).

We now show that $\left\langle W_{1}(\pi) \Psi_{0}, \mathcal{H}(\pi, \pi, 0,0) W_{1}(\pi) \Psi_{0}\right\rangle$ is close to $\left\langle\Psi_{0}, \mathcal{H} \Psi_{0}\right\rangle$, which is the desired result as by Eq. (39) it shows that $W_{1}(\pi) \Psi_{0}$ is a state for which the expectation value of the energy with Hamiltonian $\mathcal{H}_{\pi}$ is close to the expectation value of the energy of the state $\Psi_{0}$ with Hamiltonian $\mathcal{H}(0,0,0,0)$.

To show that the expectation value of the energy is close, we write $\mathcal{H}(\pi, \pi, 0,0)=\sum_{Z} h_{Z}(\pi, \pi, 0,0)$. We define $\mathcal{S}_{1}$ to be the set of points $i$ with $-L / 4+L / 4(\xi \mu+1)<x_{0}(i)<L / 4-L / 4(\xi \mu+1)$, and define $\mathcal{S}_{2}$ to be the set of points $i$ with $L / 4+L / 4(\xi \mu+1)<x_{0}(i)<3 L / 4-L / 4(\xi \mu+1)$. Note that $W_{1}$ is supported on $\mathcal{S}_{1}$ and $W_{2}$ is supported on $\mathcal{S}_{2}$ as shown in Fig. 2. If $Z \cap \mathcal{S}_{1}=\emptyset$, then $\left\langle W_{1}(\pi) \Psi_{0}, h_{Z}(\pi, \pi, 0,0) W_{1}(\pi) \Psi_{0}\right\rangle=$ $\left\langle\Psi_{0}, h_{Z} \Psi_{0}\right\rangle . \quad$ If $Z \cap \mathcal{S}_{2}=\emptyset$, then $\mid\left\langle W_{1}(\pi) \Psi_{0}, h_{Z}(\pi, \pi, 0,0) W_{1}(\pi) \Psi_{0}\right\rangle$ - 
$\left\langle\Psi_{0}, h_{Z} \Psi_{0}\right\rangle|\quad=\quad|\left\langle W_{1}(\pi) W_{2}(\pi) \Psi_{0}, h_{Z}(\pi, \pi, 0,0) W_{1}(\pi) W_{2}(\pi) \Psi_{0}\right\rangle \quad-$

$\left\langle R^{\uparrow}(\pi, X) R^{\downarrow}(-\pi, X) \Psi_{0}, h_{Z}(\pi, \pi, 0,0) R^{\uparrow}(\pi, X) R^{\downarrow}(-\pi, X) \Psi_{0}\right\rangle \mid \leq 2\left(c_{2}+c_{3}\right)\left\|h_{Z}\right\| . \quad$ Fi-

nally, the sum of $\left\|h_{Z}\right\|$ over all $Z$ such that $Z \cap \mathcal{S}_{1} \neq \emptyset$ and $Z \cap \mathcal{S}_{2} \neq \emptyset$ is bounded by $V s_{1} \exp [-\mu L / 4(\xi \mu+1)]$. Thus, we find that

$$
\begin{aligned}
& \left|\left\langle W_{1}(\pi) \Psi_{0}, \mathcal{H}(\pi, \pi, 0,0) W_{1}(\pi) \Psi_{0}\right\rangle-\left\langle\Psi_{0}, \mathcal{H} \Psi_{0}\right\rangle\right| \\
\leq & C_{7} V^{2} \sqrt{L / v \Delta E} s_{1}^{2} \exp [-(L-L / 4(\xi \mu+1)) / \xi] \\
= & C_{7} V^{2} \sqrt{L / v \Delta E} s_{1}^{2}(\exp [-L / 4(\xi+1 / \mu)]),
\end{aligned}
$$

giving Eq. (36) as claimed.

This calculation can be extended to systems with larger symmetry groups than the $U(1)$ symmetry above. If a system has $N$ species of fermions, with an $S U(N)$ symmetry, one may define angles $\theta_{1}^{a}, \theta_{2}^{a}$ for $a=1 \ldots N$ for each species of fermion. We perform a continuation with $\theta_{1}^{a}=\theta /(N-1)$ for $1 \leq a \leq N-1$, and $\theta_{1}^{N}=-\theta$, going from $\theta=0$ to $\theta=2 \pi(N-1) / N$. Then, after the continuation we have $\theta_{1}^{a}=2 \pi / N$ for $1 \leq a \leq N$, and $\theta_{1}^{N}=2 \pi / N-2 \pi$. Thus, if the ground state of the Hamiltonian with no flux is an $S U(N)$ singlet and the system has a gap to the lowest state which is not an $S U(N)$ singlet, then there is a state of the Hamiltonian with flux $2 \pi / N$ which is close in energy to the ground state of the Hamiltonian without flux. Colloquially, the flux is quantized in units of $2 \pi / N$.

\section{DISCUSSION}

We have used quasi-adiabatic continuation as a tool to study systems with continuous symmetries and a gap. This leads to a version of Goldstone's theorem for nonrelativistic systems which is valid in arbitrary finite dimension and which does not depend on additional assumptions regarding ergodicity. While this result was derived from an infinitesimal quasiadiabatic continuation, the continuation under a non-infinitesimal change in parameters was used to derive an approximate flux periodicity of systems with a spin gap. While this periodicity is realized in different ways in different systems, for example by Byers-Yang states in an $s$-wave superconductor and by topological excitations in an RVB state, the mathematical result covers both cases.

Finally, we have discussed the Berry phase under quasi-adiabatic continuation, and shown that it is close to the usual non-Abelian Berry phase for adiabatic evolution. This result 
will be used in a future work to consider Hall conductance quantization in many-body systems, and, under the assumption of an excitation gap, to remove the need for an averaging assumption on the Hall conductance[25].

Acknowledgments - I thank N. Bonesteel and T. Koma for useful discussions. This work was carried out under the auspices of the NNSA of the U.S. DOE at LANL under Contract No. DE-AC52-06NA25396.

[1] V. Kalmeyer and R. B. Laughlin, Phys. Rev. Lett. 59, 2095 (1987); N. Read and S. Sachdev, Phys. Rev. Lett. 66, 1773 (1991); X.-G. Wen, Phys. Rev. B 44, 2664 (1991).

[2] M. B. Hastings and X.-G. Wen, Phys. Rev. B 72, 045141 (2005).

[3] M. B. Hastings, Phys. Rev. B 69, 104431 (2004).

[4] M. B. Hastings, Phys. Rev. Lett. 93, 140402 (2004).

[5] M. B. Hastings and T. Koma, Commun. Math. Phys. 265, 781 (2006).

[6] B. Nachtergaele and R. Sims, Commun. Math. Phys. 265, 119 (2006).

[7] The idea of studying the flux quantization problem was suggested to the author by N. Bonesteel.

[8] N. Byers and C. N. Yang, Phys. Rev. Lett. 7, 46 (1961).

[9] S. A. Kivelson, D. S. Rokhsar, and J. P. Sethna, Europhys. Lett. 6, 353 (1988).

[10] E. H. Lieb and D. W. Robinson, Commun. Math. Phys. 28, 251 (1972).

[11] I. Affleck, T. Kennedy, E. H. Lieb, and H. Tasaki, Commun. Math. Phys. 115, 477 (1988).

[12] I. Affleck and E. H. Lieb, Lett. Math. Phys. 12, 57 (1986).

[13] T. J. Osborne, preprint quant-ph/0601019.

[14] S. Bravyi, M. B. Hastings, and F. Verstraete, Phys. Rev. Lett. 97, 050401 (2006).

[15] F. Wilczek and A. Zee, Phys. Rev. Lett. 52, 2111 (1984).

[16] T. Koma, arxiv.org math-ph/0505022.

[17] W. F. Wreszinski, Fortschr. Phys. 35, 379 (1987); L. Landau, J. Fernando Perez and W. F. Wreszinski, J. Stat. Phys. 26, 755 (1981).

[18] V. N. Kotov, O. Sushkov, Z. Weihong, and J. Oitmaa, Phys. Rev. Lett. 80, 5790 (1998).

[19] See Kaj Höglund, A. W. Sandvik, and S. Sachdev, Phys. Rev. Lett. 98, 87203 (2007) for a study of the behavior of a single such defect near a quantum critical point. 
[20] N. Alon, Combinatorica, 6(2), 83 (1986); J. Friedman, Conf. Proc. of the Annual ACM Symposium on Theory of Computing, 720 (2003); J. Friedman, preprint cs/0405020.

[21] N. W. Ashcroft and N. D. Mermin, Solid State Physics, Chapter 33, (Harcourt Brace College Publishers, New York, 1976).

[22] T. Senthil and M. P. A. Fisher, Phys. Rev. B 63, 134521 (2001).

[23] A. Paramekanti and A. Vishwanath, Phys. Rev. B 70, 245118 (2004).

[24] M. B. Hastings, Europhys. Lett. 70, 824 (2005).

[25] Q. Niu and D. J. Thouless, Phys. Rev. B 35, 2188 (1987). 